\title{
Conciliación de la medicación en farmacia comunitaria
}

\section{María José Coronado Núñez ${ }^{1}$, Estefanía Bravo Moreno², Ana Isabel Beas Morales³, Tomás Tena Trincado4, Manuel Castillo López ${ }^{5}$, Clara Alonso Larrocha ${ }^{6}$}

1. Doctora en Farmacia. Directora del Centro de Información del Medicamento. Colegio Oficial de Farmacéuticos de Huelva. 2. Farmacéutica comunitaria en Huelva. 3. Farmacéutica comunitaria en Ayamonte (Huelva). 4. Farmacéutico comunitario en Lepe (Huelva). 5. Farmacéutico comunitario en San Juan del Puerto (Huelva). 6. Farmacéutica del Centro de Información del Medicamento. Colegio Oficial de Farmacéuticos de Huelva

\section{PALABRAS CLAVE}

Conciliación, tratamiento farmacológico, prescripción inadecuada, seguridad, farmacia comunitaria

\section{KEYWORDS}

Medication reconciliation, therapeutics, inappropiate prescribing, safety, community pharmacy

\section{RESUMEN}

Introducción: La conciliación del tratamiento farmacológico consiste en revisar y registrar de forma completa y precisa la medicación habitual de un paciente, con objeto de conseguir la seguridad del mismo en cuanto al uso de los medicamentos. El objetivo principal de este estudio es cuantificar las discrepancias de conciliación producidas como consecuencia de la transición sanitaria del paciente entre los distintos niveles asistenciales.

Métodos: Estudio piloto descriptivo multicéntrico desarrollado con 29 pacientes en seis farmacias comunitarias onubenses durante tres meses. Se estudia el tratamiento farmacológico del paciente comparando su tratamiento habitual con el nuevo tratamiento instaurado. Para la toma de decisiones se emplean la Base de Datos de Medicamentos y Productos Sanitarios, el método Dáder en seguimiento farmacoterapéutico y los criterios Stopp-Start en pacientes mayores de 65 años.

Resultados: En un 37,9\% de los pacientes estudiados se encontraron discrepancias en la medicación, principalmente en pacientes que proceden del hospital (36,4\% tras alta hospitalaria y $45,5 \%$ tras visita al especialista).

Las discrepancias mayoritarias tras la conciliación se detectan en la patología cardiovascular, seguida en menor medida por la patología renal. En un 36,4\% de los casos el tipo de error observado ha sido la duplicidad de tratamiento farmacológico para una misma patología y en un 27,3\% de los pacientes se detectaron enfermedades que no estaban siendo tratadas. Asimismo, se observaron pautas erróneas en el tratamiento $(18,2 \%)$ y dosis erróneas de administración (9,1\%). Un 81,8 \% de los pacientes fue derivado al médico.

Conclusión: Las discrepancias en el tratamiento farmacológico de un paciente tras el cambio de nivel asistencial son muy elevadas. Dado que el farmacéutico comunitario es el último profesional sanitario en contacto con el paciente antes de la administración del medicamento, su intervención permite detectar estas discrepancias, disminuyendo la morbilidad asociada a las mismas y contribuyendo a garantizar la continuidad asistencial.

\section{Reconciliation of the medication in community pharmacy}

\section{ABSTRACT}

Introduction: Proper usage of medicines is the main objective of the medication reconciliation. It consists of a method to review systematically the patient medicine intakes to guarantee their safety.

Objectives: This paper aims to demonstrate the correlation between failure in treatment reconciliation and patient changes in the different care levels.

Methods: A multisite descriptive pilot study was developed in six pharmacies from Huelva for three months. Twenty nine patients' ordinary pharmacological treatments were compared to new prescribed treatments. BOT PLUS medicine database, Dader method and Stopp-Start criteria were used in the decision making process to analyze data.

Results: A 37.9\% patients show discrepancies in their pharmacological treatments, mainly those who come from a hospital discharge (36.4\%) and those who come from specialists (45.5\%).

Heart disease patients showed the highest discrepancies, followed by kidney disease patients. Discrepancies were due to double prescription for the same pathology (36.4\%) and non treatment diseases detection (27.3\%). Additionally, mistakes in treatment (18.2\%) and mistakes in dosage prescription $(9.1 \%)$ were observed. As a consequence, $81.8 \%$ were sent back to receive medical attention.

Discussion: As a summary, discrepancies in pharmacological treatments are found to be very high when patients change care levels. Pharmacist role to avoid these discrepancies is key, since they are the last sanitary professional in contact with the patient before medicines intake.

Premio a la mejor comunicación en la categoría de seguimiento farmacoterapéutico en el VI Congreso Nacional de Farmacéuticos Comunitarios. SEFAC. Málaga 22-24 mayo 2014.

Financiación: Ninguna ajerna.

Conflicto de intereses: Los autores declaran no existir conflicto de intereses en relación con el contenido del artículo.

Cite este artículo como: Coronado MJ, Bravo E, Beas Al, Tena T, Castillo M, Alonso C. Conciliación de la medicación en farmacia comunitaria. Farmacéuticos Comunitarios. 2015 Sep 01; 7(3):19-22. doi:10.5672/FC.2173-9218.(2015/Nol7).003.04

Autora para correspondencia: Ma José Coronado Núñez (cimhuelva@redfarma.org).

ISSN 1885-8619 @SEFAC (Sociedad Española de Farmacia Familiar y Comunitaria). Todos los derechos reservados. 


\section{Introducción}

La conciliación del tratamiento farmacológico consiste en revisar y registrar de forma completa y precisa la medicación habitual de un paciente, con objeto de conseguir la seguridad del mismo en cuanto al uso de los medicamentos (1). Por ello, es de vital importancia coordinar las enfermedades del paciente y su medicación habitual con el tratamiento farmacológico que se añade durante las distintas transiciones asistenciales como son los ingresos en el hospital, traslados intrahospitalarios, altas hospitalarias o asistencia en urgencias.

Desafortunadamente esta actividad no se realiza de forma rutinaria y continua a lo largo de todo el proceso asistencial y para todos los pacientes atendidos, lo cual es causa de un elevado número de errores que pueden ser motivos de reingresos hospitalarios no planificados y que suponen costes económicos prevenibles (2).

Aunque un 66\% de las notificaciones de errores de conciliación corresponden a transiciones intrahospitalarias, el número de discrepancias farmacológicas por paciente suele ser más elevado durante los procesos de alta hospitalaria (3). Hay estudios que ponen de manifiesto que hasta un $12 \%$ de los pacientes experimenta efectos adversos debido a su tratamiento en las dos semanas posteriores al alta hospitalaria y en un 4\% de los casos puede dar lugar a efectos graves sobre el paciente (3). Así pues, es importante evitar las discrepancias en el tratamiento farmacológico en cualquier transición asistencial pero especialmente tras el alta hospitalaria, ya que la perpetuación de estos errores puede dar lugar a interacciones, duplicidades $u$ otros efectos no deseados sobre el paciente. Existen además, poblaciones más sensibles a estas posibles discrepancias o errores farmacológicos, como es el caso de los pacientes polimedicados o aquellos pacientes que toman medicamentos que no requieren receta médica (1).

Por todo lo expuesto, y a pesar de que en su desarrollo inicial la conciliación de los tratamientos farmacológicos de un paciente se limitó a centros hospitalarios y tenía como principales protagonistas a los farmacéuticos clínicos, una visión amplia del concepto implica también a la atención primaria y a otros profesionales como médicos de familia y farmacéuticos comunitarios. Desde la farmacia comunitaria se puede contribuir a minimizar los errores derivados de la ausencia de conciliación de los tratamientos farmacológicos, disminuyendo así la morbilidad asociada a ellos y contribuyendo a garantizar la continuidad asistencial y a reducir los costes económicos.

\section{Objetivos}

Objetivo principal

Cuantificar las discrepancias de conciliación producidas como consecuencia de la transición sanitaria del paciente entre los distintos niveles asistenciales.

\section{Objetivos secundarios}

- Detectar y tipificar posibles errores de conciliación en el paciente y clasificarlos en función de la patología implicada.

- Promover la comunicación entre los profesionales de la salud de los distintos niveles asistenciales (médicos de atención primaria y especializada y farmacéutico comunitario).

\section{Material y métodos}

Estudio piloto descriptivo multicéntrico desarrollado en seis farmacias comunitarias onubenses durante tres meses (febrero-abril 2014).

Se estudió la medicación de un total de 29 pacientes que cumplían los siguientes criterios de inclusión:

Paciente con tratamiento crónico que acude a la farmacia a retirar medicamentos tras un alta hospitalaria, tras la visita a un profesional sanitario del ámbito público o privado, o en cualquier otra situación en la que se instaure un nuevo tratamiento o se modifique su tratamiento habitual.

Paciente para el que sea posible acceder a su historial farmacoterapéutico, así como a los nuevos tratamientos instaurados o cambios establecidos en su medicación (informe médico).

Se elaboró un estado de situación adaptado según el método Dáder (4), en el que se reflejan, por un lado, los medicamentos que habitualmente retira el paciente (en base al historial de dispensaciones del programa de gestión de la farmacia y de la aplicación de Receta XXI), incluyéndose también los medicamentos que utiliza el paciente sin receta médica; por otro lado, se han incluido los nuevos tratamientos instaurados tras un alta hospitalaria o una visita a un profesional sanitario del ámbito público o privado, o cualquier otra situación en la que se ha instaurado un nuevo tratamiento o se ha modificado su tratamiento habitual.

Se ofreció el estudio a los pacientes que acuden a la farmacia y, cumpliendo los criterios de inclusión, acceden verbalmente a participar en el mismo.

Se registraron las posibles discrepancias de medicación y en los casos necesarios el paciente ha sido derivado a su médico de familia.

Para la toma de decisiones del estado de situación del paciente, se ha utilizado la Base de Datos de Medicamentos y Productos Sanitarios del Consejo General de Colegios Oficiales de Farmacéuticos (Bot Plus) (4), el método Dáder en seguimiento farmacéutico (5) así como en los criterios Stopp-Start, en el caso de pacientes mayores de 65 años (6). El tratamiento estadístico de los datos se ha llevado a cabo mediante el programa informático SPSS ${ }^{\circledR}$.

\section{Resultados}

Del total de 29 pacientes estudiados en las farmacias comunitarias onubenses, 12 pacientes $(41,4 \%)$ proceden de un alta hospitalaria, 10 pacientes $(34,5 \%)$ del especialista público, 4 pacientes $(13,8 \%)$ del especialista privado y 3 pacientes (10,3\%) de urgencias. Tal y como muestra la figura 1 , en 11 casos $(37,9 \%)$ del total de casos estudiados se encontraron discrepancias en la medicación, principalmente en pacientes que proceden del hospital $(36,4 \%$ tras alta hospitalaria y $45,5 \%$ tras visita al especialista de ámbito público).

Las discrepancias mayoritarias tras la conciliación se detectan en la patología cardiovascular, seguida en menor medida por la patología renal (figuras 2 y 3). Tal y como muestra la figura 4 , en 4 casos $(36,4 \%)$ el tipo de error observado ha sido la duplicidad de medicación para una misma patología y en 3 pacientes $(27,3 \%)$ se detectaron enfermedades que no estaban siendo tratadas. Asimismo, se observaron pautas erróneas en el tratamiento en 2 pacientes $(18,2 \%) \mathrm{y}$ 
dosis erróneas de administración en un caso $(9,1 \%)$.

9 pacientes $(81,8 \%)$ fueron derivados al médico sin que se disponga de datos sobre el resultado de la derivación.

\section{Discusión}

La conciliación farmacéutica de los tratamientos debe convertirse en un servicio necesario para detectar discrepancias y evitar errores y problemas relacionados con la medicación. Como se puede observar en este trabajo, las discrepancias en el tratamiento farmacológico de un paciente que requieren aclaración tras el cambio de nivel asistencial son muy elevadas, resultados que concuerdan con estudios previos en este sentido $(6,15,16)$.

Los medicamentos que más discrepancias originan son los agentes cardiovasculares y las discrepancias de conciliación más frecuentes son las duplicidades y omisión de medicamentos, al igual que ponen de manifiesto estudios anteriores $(1,13,15,16)$. Sólo el $28 \%$ de los errores de conciliación que se producen tras un alta hospitalaria son interceptados antes de que lleguen al paciente (7), pudiendo ocasionar consecuencias potencialmente más graves.

Si bien este estudio es un estudio piloto, llevado a cabo con un número limitado de pacientes, sirve para poner de manifiesto la importancia de la conciliación de la medicación. En la sociedad actual, una población cada vez más envejecida, con enfermedades crónicas de elevada complejidad, la conciliación de la medicación no es una actividad prescindible sino una necesidad cuya responsabilidad se debe compartir entre todos los profesionales sanitarios, implicados, sin olvidar al propio paciente y a sus familiares. El farmacéutico comunitario es el último profesional sanitario en contacto con el paciente antes de la administración del medicamento. Por este motivo, entre otros, la conciliación desde la farmacia comunitaria debe considerarse prioritaria destacándose como el lugar ideal para detectar discrepancias y comunicarlas. El desconocimiento del resultado de las intervenciones del médico ante las derivaciones del farmacéutico indica que sería necesario establecer un sistema de comunicación adecuado y

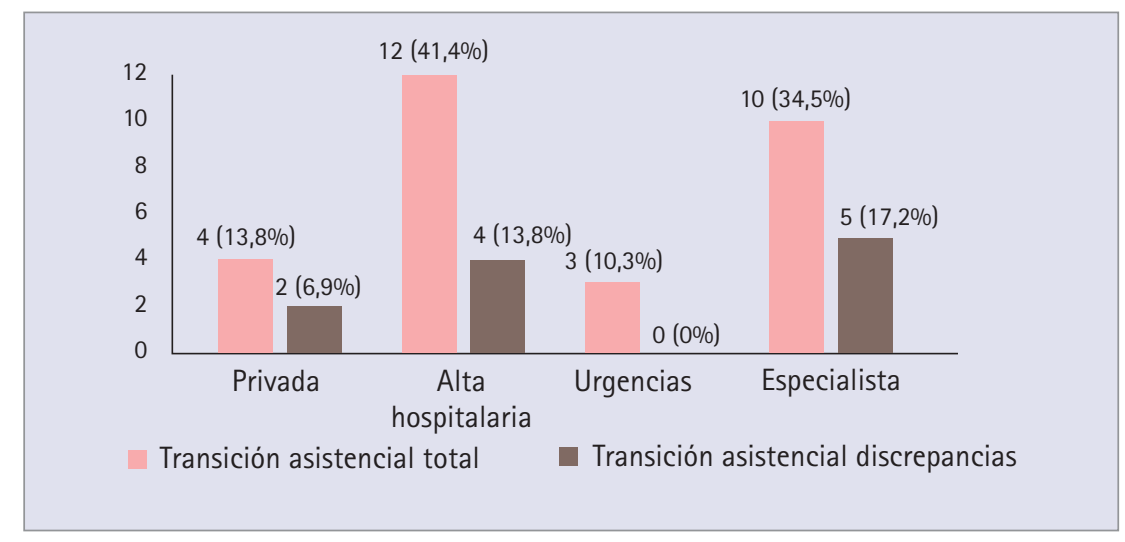

Figura 1. Tipo de transición asistencial estudiado

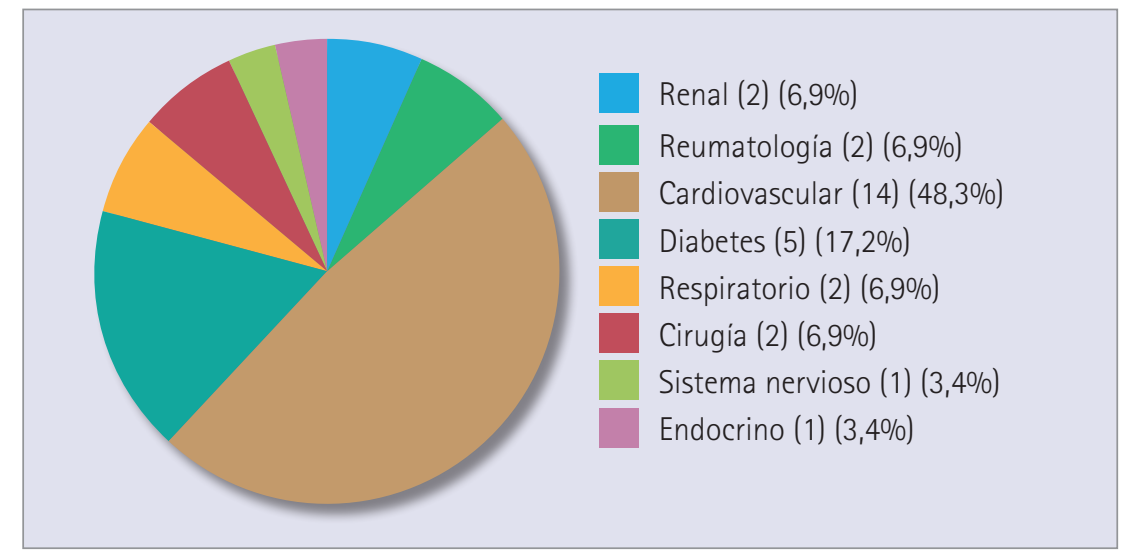

Figura 2. Patologías implicadas en el total de casos estudiados

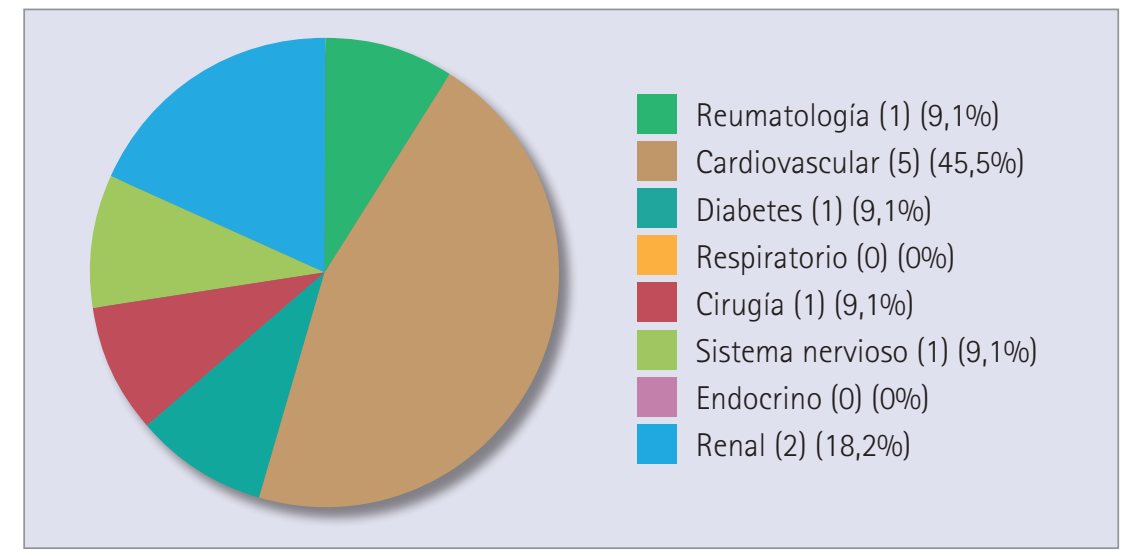

Figura 3. Patologías implicadas en los casos con discrepancias detectadas

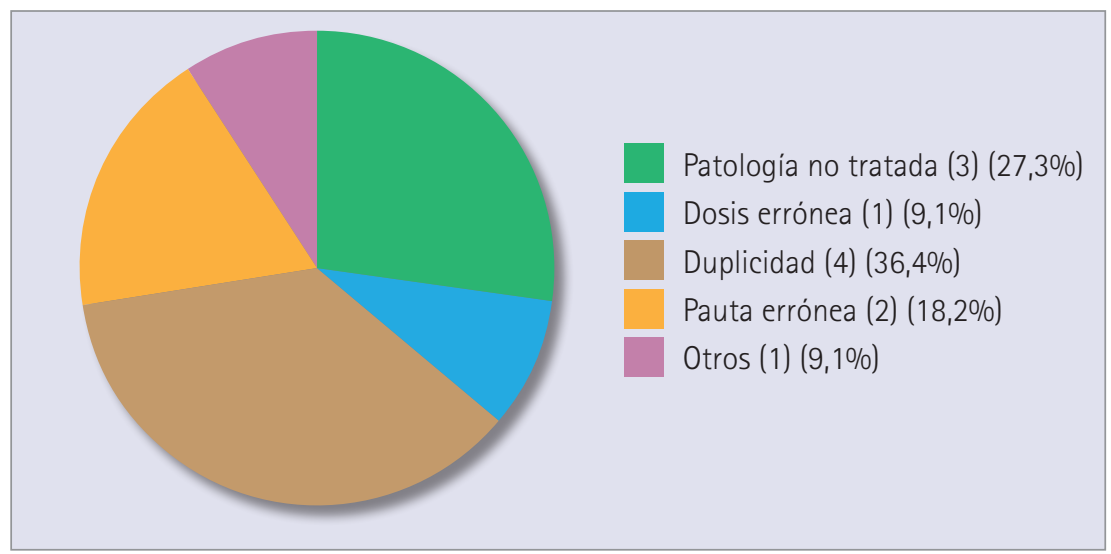

Figura 4. Tipos de discrepancias detectadas 
rápido farmacéutico-médico con objeto de facilitar esta tarea y contribuir a disminuir la morbilidad asociada a este tipo de situaciones, garantizándose la continuidad asistencial.

\section{Agradecimientos}

Lcda. Ana Rivera Bocanegra y Lcda. María Isabel Gómez Bernal, farmacéuticas comunitarias, por su colaboración en la recopilación de casos clínicos.

Lcda. Rosa Barba Márquez, farmacéutica comunitaria, por su colaboración en la explotación de los resultados.

\section{Referencias bibliográficas}

1. Quiñones C. Conciliación del Tratamiento Farmacológico. En: Formación Continuada para farmacéuticos de Hospital V. Gerona: Hospital de Figueres, Fundación Salud Empordá; 2012. Págs. 97-177.

2. Juvany R, Jódar R. Importancia de la conciliación de la medicación para garantizar la continuidad y la seguridad del proceso asistencial. Med Clin. 2012; 139: 672-673. doi.or$\mathrm{g} / 10.1016 / j . m e d c l i .2012 .07 .010$

3. Bravo R, Caballero N [Internet]. Conciliación de la medicación en el paciente polimedicado. En: Curso Polimedicación y Salud. Estrategias para el abordaje integral de los pacientes polimedicados. Laboratorio de Prácticas Innovadoras en Polimedicación y Salud. [Acceso 21/10/2014]. Disponible en: http://www.polimedicado.com/
4. Sabater D, Silva MM, Faus MJ. Guía de Seguimiento Farmacoterapéutico $3^{\text {a }}$ Ed. Grupo de Investigación en Atención Farmacéutica. Granada: Universidad de Granada GIAF-UGR (CTS-131); 2007.

5. Consejo General de Colegios Oficiales de Farmacéutico. Base de datos de Medicamentos y Productos Sanitarios. Actualización abril 2014. Madrid: CGCOF; 2014.

6. Delgado E, Muñoz M, Montero B, Sánchez C, Gallagher P, Cruz-Jentoft A. Prescripción inapropiada de los medicamentos en los pacientes mayores: los criterios STOPP/START. Rev Esp Geriatr Gerontol, 2009;44(5):273-279. doi.org/10.1016/j.regg.2009.03.017

7. Roure C, Queralt M, Delgado 0. Guía para la Implantación de Programas de Conciliación de la Medicación en los Centros Sanitarios. Barcelona: Sociedad Catalana de Farmacia Clínica; 2010.

8. Grupo de trabajo: Buenas prácticas en el uso de los medicamentos [Internet]. Buenas prácticas en la conciliación de la medicación en el ingreso, alta y transición interservicios. Observatorio para la seguridad del paciente. Junta de Andalucía. Consejería de Salud. [Acceso 12/9/2014]. Disponible en: http://www.noble-arp.com/src/ img_up/28122012.2.pdf

9. Amado E, Durán C, Izco N, Massot M, Palma D, Rodríguez G et al [Internet]. Elementos básicos del abordaje de la medicación en el paciente crónico: información al paciente, conciliación, revisión y adherencia. SEFAP. [Acceso 15/9/2014]. Disponible en: http:// issuu.com/sefap/docs/sefap/1

10. Galván-Banqueri $\mathrm{M}$, Santos-Ramos B, Vega-Cocab MD, Alfaro-Lara ER,
Nieto-Martín MD, Pérez-Guerrero C, Adecuación del tratamiento farmacológico en pacientes pluripatológicos. Atencion primaria 2013;45(1):6-20. doi.org/10.1016/j.aprim.2012.03.011

11. Delgado 0, Martinez M, Crespí M, Serra G. Conciliación de la medicación: asumamos la responsabilidad compartida. Farm.Hosp 2008;32(2):63-64. doi:10.1016/S1130-6343(08)72815-0

12. Coll de Tuero G. Revisión de la medicación en ancianos polimedicados. Aten Primaria 2012;44(8):461-462 doi:10.1016/j.aprim.2012.06.008

13. Geurts MM, Talsma J, Brouwers JR, de Gier JJ. Medication review and reconciliation with cooperation between pharmacist and general practitioner and the benefit for the patient: a systematic review. Br J Clin Pharmacol 2012;74(1):16-33. doi:10.1111/j.13652125.2012.04178. $\mathrm{x}$

14. Alfaro-Lara ER, Vega-Cocab MD, Galván-Banqueri M, Nieto-Martín MD, Pérez-Guerrero C, Santos-Ramos B. Metodología de conciliación del tratamiento farmacológico en pacientes pluripatológicos. Aten Primaria 2014;46(2):89-99. doi:10.1016/j. aprim.2013.07.002

15. Rosich I [Internet]. Conciliación y revisión de la medicación. En: Programa Científico: IV Congreso Nacional de Atención Sanitaria al Paciente Crónico. Alicante. 8-10 de marzo 2012. [Acceso 15/9/2014]. Disponible en: http://www.ugr.es/ cts131/esp/guias/ GUIA\%20FINAL\%20DADER.pdf

16. López-Martín C, Aquerreta I, Faus V, Idoate A. Conciliación de la medicación en el paciente crítico. Med Intensiva 2014; 38(5): 283-287. doi: 10.1016/j.medin.2013.04.008 